\title{
(6) OPEN ACCESS \\ MRI in Leber's hereditary optic neuropathy: the relationship to multiple sclerosis
}

\author{
Lucy Matthews, ${ }^{1,2}$ Christian Enzinger, ${ }^{3,4}$ Franz Fazekas, ${ }^{3}$ Alex Rovira, ${ }^{5}$ \\ Olga Ciccarelli, ${ }^{6}$ Maria Teresa Dotti, ${ }^{7}$ Massimo Filippi, ${ }^{8}$ Jette L Frederiksen, ${ }^{9}$ \\ Antonio Giorgio, ${ }^{7}$ Wilhelm Küker, ${ }^{1,2}$ Carsten Lukas, ${ }^{10}$ Maria A Rocca, ${ }^{8}$ \\ Nicola De Stefano, ${ }^{7}$ Ahmed Toosy, ${ }^{6}$ Tarek Yousry, ${ }^{6}$ Jacqueline Palace, ${ }^{1,2}$ On behalf of \\ the MAGNIMS network
}

\begin{abstract}
- Additional material is published online only. To view please visit the journal online (http://dx.doi.org/10.1136/ jnnp-2014-308186)

For numbered affiliations see end of article.
\end{abstract}

\section{Correspondence to} Dr Jacqueline Palace, Department of Neurology, Nuffield Department of Clinical Neurosciences, Oxford University Hospitals NHS Trust, University of Oxford, Level 3 John Radcliffe Hospital, Oxford, OX3 9DU, UK; jacqueline.palace@ndcn.ox.ac. uk

Received 25 March 2014 Revised 28 May 2014 Accepted 18 June 2014 Published Online First 22 July 2014

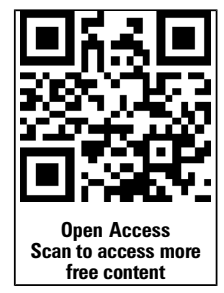

CrossMark

To cite: Matthews $L$, Enzinger $C$, Fazekas $F$, et al. J Neurol Neurosurg Psychiatry 2015;86: 537-542.

\section{ABSTRACT}

Background Leber's hereditary optic neuropathy (LHON) and a multiple sclerosis (MS)-like illness appear to coexist 50 times more frequently than would be expected by chance. This association of LHON and MS (LMS) raises an important question about whether there could be a common pathophysiological mechanism involving mitochondrial dysfunction.

Objective The primary aim was to define MRI features of LMS and LHON, and to assess the proportions of individuals displaying features typical of MS. Secondarily, we investigated the effect of gender on the risk of developing white matter lesions in the context of LHON.

Methods A blinded standardised review of conventional brain MRIs of 30 patients with MS, 31 patients with LHON and 11 patients with LMS was conducted by three independent experts in the field. MS-like MRI features were assessed.

Results All patients with LMS and $26 \%$ of patients with LHON had white matter lesions. Of these, all patients with LMS and $25 \%$ with LHON were found to have an MRI appearance typical of MS. Female patients with LHON had a significantly greater risk of having white matter lesions consistent with MS compared with male patients (relative risk 8.3).

Conclusions A blinded review of conventional brain MRIs shows that patients with LMS have a scan appearance indistinguishable from MS. Mitochondrial dysfunction could be a common pathophysiological pathway in the formation of white matter lesions. There appears to be a strong female influence on the radiological appearance as well as clinical development of MS in patients with LHON.

\section{INTRODUCTION}

Leber's hereditary optic neuropathy (LHON) is a maternally inherited mitochondrial condition that manifests as painless subacute bilateral visual loss usually in early adulthood. It is caused by DNA point mutations at sites 3460, 11778 and 14484 that encode complex 1 of the respiratory chain. A multiple sclerosis (MS)-like disease in association with LHON (LMS) is very rare but coexists about 50 times more frequently than expected by chance. ${ }^{1-4}$ This may suggest that mitochondrial disturbance is important in the pathophysiology of MS.

The association between LHON and MS was first described by Lees $e t a l^{5}$ in 1964. A larger case series was published by Harding et al, ${ }^{1}$ and reported 11 patients with LHON and MS. ${ }^{6}$ Thus, the association is often referred to as 'Harding's disease'. Although LHON shows a far greater penetrance in men (approximately $77 \%$ of cases), ${ }^{7}$ only a third of the patients with Harding's disease are men. ${ }^{2}$ It has been theorised that when the high threshold for clinical expression in women with Leber's mutations is crossed, MS pathology may be triggered. ${ }^{2}$

MS is associated with distinctive MRI features. ${ }^{8}$ Therefore, if the imaging characteristics of Harding's disease are indistinguishable from those of MS, this would provide further support for a common pathophysiological mechanism. Of note, a previous report of two cases of Harding's disease suggested that there are important differences, ${ }^{9}$ with reduced brightness and indistinct margins of T2 lesions and a lack of T1 hypointensity on MRIs. However, other patients with more typical appearances have also been reported in the literature. ${ }^{6} 10$

The objectives of this study were to assess whether the MRI appearances of patients with Harding's disease (LMS) differ from those with relapsing remitting MS, to characterise cerebral MRI findings in LHON and, more specifically, to determine whether female patients with LHON are more likely to have brain MS-like lesions than male patients.

\section{METHODS}

Data collection

Brain MRIs were retrospectively obtained from six sites (Oxford, Milan, Siena, London, Bochum and Copenhagen) from patients with clinical LHON with or without a relapsing MS-like illness, and patients with age-matched relapsing MS for comparison. Thirty-one patients with LHON and 11 with an MS-like illness (LMS) were identified, and 30 MS controls were selected. Participant characteristics are summarised in table 1.

Patients with MS and LMS required two or more relapses disseminated in time and space. All patients with LHON and LMS had a confirmed genetic mutation. Patients with LMS were diagnosed locally in MS specialist centres and had clinical histories consistent with MS. Patients with LHON had been scanned either routinely or for research purposes. The imaging data for each 
Table 1 Participant characteristics

\begin{tabular}{lllll}
\hline & & LHON & LMS & MS \\
\hline Number & 31 & 11 & 30 \\
Age at time of MRI, years, range & & $19-50$ & $33-61$ & $22-65$ \\
Sex & & $27 \mathrm{M} / 4 \mathrm{~F}$ & $3 \mathrm{M} / 8 \mathrm{~F}$ & $10 \mathrm{M} / 20 \mathrm{~F}$ \\
Genetic mutation & m.3460G $>\mathrm{A}$ & 3 & 1 & $\mathrm{NA}$ \\
& $\mathrm{m} .11778 \mathrm{G}>\mathrm{A}$ & 23 & 8 & $\mathrm{NA}$ \\
& $\mathrm{m} .14484 \mathrm{~T}>\mathrm{C}$ & 5 & 2 & $\mathrm{NA}$ \\
\hline
\end{tabular}

LHON, Leber's hereditary optic neuropathy; LMS, MS-like disease in association with LHON; MS, multiple sclerosis; NA, not applicable; WMLs, white matter lesions visible on T2 MRI.
Table 2 Summary of results

\begin{tabular}{|c|c|c|c|}
\hline & LHON & LMS & MS \\
\hline Total & 31 & 11 & 30 \\
\hline T2 lesions & $8(26 \%)$ & $11(100 \%)$ & $30(100 \%)$ \\
\hline T1 hypointense lesions & $4(13 \%)$ & $9(82 \%)$ & $23(77 \%)$ \\
\hline Brain atrophy & $4(13 \%)$ & $5(45 \%)$ & $13(43 \%)$ \\
\hline $\begin{array}{l}\text { MRI consistent with MS as judged by at } \\
\text { least two of three raters }\end{array}$ & $2(6.5 \%)$ & $11(100 \%)$ & $27(90 \%)$ \\
\hline $\begin{array}{l}\text { Modified McDonald's dissemination in } \\
\text { space criteria }\end{array}$ & $2(6.5 \%)$ & $8(73 \%)$ & $30(100 \%)$ \\
\hline
\end{tabular}

participant included good quality 1.5 Tesla transverse T2-weighted and unenhanced T1-weighted brain images as a minimum. Participants with a neurological disease that would change the appearance of the brain MRI were excluded. All the five patients with LMS for whom we had the results of cerebrospinal fluid examination were positive for unmatched oligoclonal bands.

All the MRI and clinical data sets were first collated, anonymised and stored at the contributing sites in accordance with the local research ethics regulations.

\section{Scan collation and scoring}

The imaging and clinical data were collated centrally in Oxford, quality controlled and blinded (by LM). The brain MRIs for all participants were then independently reviewed by three experts in the field of MRI and MS (FF, CE and AR), blinded to clinical and demographic data. The scoring criteria had been preagreed by the Magnetic Resonance Imaging in MS (MAGNIMS) collaborative group and required the reviewers to first comment on whether the scans were normal or abnormal, and then to document the features of the scan including the location, symmetry, morphology, border and size of T2 lesions. T1 hypointense lesion characteristics were also recorded including their presence, size and location. The presence of brain atrophy was assessed by visual inspection (ie, quantitative volumetric analysis was not performed). The expert reviewers were then asked to comment on whether they considered MRI as being consistent with MS. The questionnaire is provided in the online supplementary material as eFigure 1.

\section{Data analysis}

The results of the questionnaires were compiled and unblinded. The inter-rater agreement between the three independent reviewers was calculated using Fleiss' $\kappa$.

An adapted version of the 2010 revision to McDonald's MRI criteria regarding 'dissemination in space', to take into account the lack of spinal cord imaging, was applied to each participant's brain MRI. This criterion is the presence of at least one T2 lesion in two of three locations (periventricular, juxtacortical or infratentorial) and is based on the work of Swanton et al. ${ }^{11}$

\section{RESULTS}

All patients with MS and LMS and 8 of 31 of those with LHON had T2 hyperintense white matter lesions (table 2).

The inter-rater agreement for whether a scan was considered to be typical of MS was high at 94\%, $\kappa=0.88, \mathrm{p}<0.001$. MRIs of 27 of $30(90 \%)$ patients with MS were rated as consistent with MS by all three reviewers. Two of the three non-consistent MS scans had complete agreement and one was rated as such by two of the three reviewers. These three patients had a relatively benign clinical course of MS with two relapses only. Two of 8 with MS as shown in table 3. All the patients with LMS were thought to have scans consistent with MS by at least two of the three reviewers.

The characteristics of the lesions were similar in patients with MS and LMS but patients with LHON and T2 hyperintense white matter lesions had notably less oval lesions and no Dawson's fingers or diffuse type lesions (ie, ill-defined widespread lesions; figure 1A). Seven of eight patients with LHON had small lesions between 2 and $5 \mathrm{~mm}$ in size, and one had a lesion of $15 \mathrm{~mm}$. The patients with LMS and MS generally had larger lesions ranging from 2 to $25 \mathrm{~mm}$.

T2 lesions (figure 1B) had a similar distribution in MS and LMS, whereas in the LHON group there were proportionally fewer lesions in the corpus callosum and none in the cerebellum.

T1 hypointensity was found in the majority of patients with MS and LMS (table 2), but only in three of the eight patients with LHON and an abnormal MRI. Brain MRIs with features of atrophy were similarly common in patients with MS and LMS (table 2).

Figure 2 shows some illustrative brain MRIs of patients with LMS and LHON with T2 hyperintense white matter lesions. A detailed overview of the patients with LHON and LMS is shown in table 3 .

In total, $90 \%$ of patients with MS, $6.7 \%$ of patients with LHON and $73 \%$ of patients with LMS fulfilled McDonald's criterion for the dissemination of brain lesions in space, as specified in the Methods section. There were no clear differences in lesion location or morphology between genetic mutations. However, the number of patients with $3460 \mathrm{G}$ and 14484T mutations was relatively small. There were no remarkable comments in the 'other comments' section of the scoring questionnaire.

Of the 12 women in total with LHON, 8 had LMS and 3 had asymptomatic T2 hyperintense white matter lesions. Of the 30 men in total with LHON, 3 had LMS and 5 had asymptomatic T2 hyperintense white matter lesions. None of the scans of the five men with LHON alone but two of the three women with LHON with T2 hyperintense white matter lesions were felt to be MS consistent (and one reviewer scored the remaining women as being consistent). Thus, the increased relative risk of women with LHON having white matter lesions typical of MS was 8.3 (95\% CI 2.8 to $25.1, \mathrm{p}<0.01)$. (25\%) abnormal MRIs in patients with LHON were consistent 


\begin{tabular}{|c|c|c|c|c|c|c|c|c|}
\hline \multirow[b]{3}{*}{ Diagnosis } & \multirow[b]{3}{*}{ Sex } & \multirow[b]{3}{*}{ Age } & \multirow[b]{3}{*}{ Genetic mutation } & \multirow{3}{*}{$\begin{array}{l}\text { Number of relapses } \\
\text { (increased optic neuritis) }\end{array}$} & \multirow{3}{*}{$\begin{array}{l}\text { Number of T2 white matter } \\
\text { lesions on MRI }\end{array}$} & \multirow{2}{*}{\multicolumn{3}{|c|}{$\begin{array}{l}\text { MRI consistent with } \\
\text { MS } \\
\text { Rater }\end{array}$}} \\
\hline & & & & & & & & \\
\hline & & & & & & 1 & 2 & 3 \\
\hline LMS & M & 42 & 14484 & $>2$ & 19 & Y & Y & $\mathrm{N}$ \\
\hline LMS & $\mathrm{F}$ & 41 & 11778 & $>2$ & $>20$ with confluence & $Y$ & $Y$ & Y \\
\hline LMS & M & 36 & 11778 & $>2$ & 1 (Confluent) & Y & $\mathrm{Y}$ & Y \\
\hline LMS & M & 45 & 11778 & $>2$ & 3 & Y & $\mathrm{Y}$ & Y \\
\hline LMS & $\mathrm{F}$ & 61 & 11778 & $>2$ & 2 & $\mathrm{Y}$ & $\mathrm{N}$ & $\mathrm{Y}$ \\
\hline LMS & $\mathrm{F}$ & 36 & 11778 & 3 with secondary progression & $>20$ & $Y$ & $Y$ & Y \\
\hline LMS & $\mathrm{F}$ & 50 & 3460 & 4 & 1 (Confluent) & Y & $Y$ & Y \\
\hline LMS & $\mathrm{F}$ & 34 & 11484 & 3 with secondary progression & 12 & Y & Y & Y \\
\hline LMS & $\mathrm{F}$ & 48 & 11778 & 4 & 2 & $Y$ & $\mathrm{~N}$ & $Y$ \\
\hline LMS & $\mathrm{F}$ & 33 & 11778 & 2 & $>20$ & $Y$ & $Y$ & $Y$ \\
\hline LMS & $\mathrm{F}$ & 32 & 11778 & 2 & $>15$ & $Y$ & $Y$ & $Y$ \\
\hline LHON & $\mathrm{F}$ & 33 & 3460 & NA & 12 & $Y$ & $Y$ & $Y$ \\
\hline LHON & $\mathrm{F}$ & 41 & 11778 & NA & $>20$ & $Y$ & $Y$ & $Y$ \\
\hline LHON & $F$ & 46 & 11778 & NA & 3 & $\mathrm{~N}$ & $N$ & $Y$ \\
\hline LHON & $M$ & 40 & 11778 & NA & 2 & $\mathrm{~N}$ & $N$ & $\mathrm{~N}$ \\
\hline LHON & $M$ & 50 & 11778 & NA & 8 & $\mathrm{~N}$ & $\mathrm{~N}$ & $\mathrm{~N}$ \\
\hline LHON & $M$ & 22 & 11778 & NA & 4 & $\mathrm{~N}$ & $\mathrm{~N}$ & $\mathrm{~N}$ \\
\hline LHON & $M$ & 68 & 11778 & NA & 6 & $\mathrm{~N}$ & $\mathrm{~N}$ & $\mathrm{~N}$ \\
\hline LHON & $M$ & 25 & 11778 & NA & 10 & $\mathrm{~N}$ & $N$ & $\mathrm{~N}$ \\
\hline
\end{tabular}

LHON, Leber's hereditary optic neuropathy; LMS, MS-like disease in association with LHON; MS, multiple sclerosis.

\section{DISCUSSION}

The findings of our study suggest that conventional MRI characteristics of MS and LMS lesions are indistinguishable to the expert eye. The morphology and location of cerebral lesions are similar, and T1 hypointense lesions and brain atrophy exist in both conditions, supporting the idea that the MRI changes in LMS are due to MS pathology and not a separate Leber's mitochondrial-related process. This is further backed by the observation that the majority of males with LHON without an MS-like illness had either normal brain MRIs or non-specific white matter lesions. In addition, our data support a strong influence of female gender on developing MS and on having radiological appearances of MS in individuals with LHON alone despite LHON being more common in men.

In accordance with LMS having MS pathology is the fact that the extraoptic MS-like disease that affects patients with LHON has the same clinical features with relapses that remit and secondary progression. ${ }^{1} 25$ Additionally, a single postmortem case report of a patient with LMS found features of MS pathology with demyelinated plaques as well as mitochondrial damage. ${ }^{12}$ The female predisposition is also similar in MS and LMS despite LHON being predominant in men.

Previous reports of the MRI appearances gave conflicting results. A case series of two patients commented on features atypical of MS. $^{9}$ They noted that their periventricular T2 lesions were either larger $(>20 \mathrm{~mm})$ or smaller $(<5 \mathrm{~mm})$ than typical Dawson's fingers, and that there were lesions with indistinct margins. T1 hypointense lesions and contrast enhancement were not found in these two cases. Features more typical of MS have been reported by Harding et $a l^{1}$ in a case series of five patients. Additionally, a quantitative MRI study of patients with LHON found abnormalities of the normal-appearing brain tissue consistent with that previously reported in MS. ${ }^{10}$ It is possible that the non-MS-like features reported by some studies might either be due to the Leber's mitochondrial pathology coexisting or these mitochondrial defects influencing the MS appearances.

A hypothesis as to why LHON may be a risk factor for MS is that mitochondrial defects trigger the autoimmune process. A further possibility is that mitochondrial dysfunction is a final common pathway in neural damage. There is an increasing body of work that suggests that mitochondrial dysfunction with subsequent energy failure is important in MS lesion formation, ${ }^{13}{ }^{14}$ axonal damage ${ }^{15}{ }^{16}$ and neurodegeneration. ${ }^{17}$ There is also some evidence that variation in mitochondrial DNA and nuclear-encoded mitochondrial genes may affect genetic susceptibility to MS. ${ }^{18}$ However, there does not appear to be a specific mitochondrial mutation (as found in LHON) or deletion in cases of MS with apparent maternal inheritance. $^{19}$

Two studies have shown that MS and clinical LHON occur more frequently than expected by chance. The first by Vanopdenbosch et $a l^{3}$ identified five patients with MS from a LHON pedigree study of 103 patients. A second paper reported 5 from 29 patients with LHON who had an MS-like illness. ${ }^{4}$ In contrast, a recent third study concluded that these conditions did not occur more frequently than expected by chance. However, there were a number of reasons why the authors may have reached an erroneous conclusion. ${ }^{20}$ The background prevalence of MS quoted was extremely high and was in fact a future 20-40-year projected value. ${ }^{21}$ The authors also did not distinguish between the higher rate of LHON mutations versus clinical LHON; penetrance is only $50 \%$ in men and $14 \%$ in women. $^{7}$ In addition, this study used a voluntary surveillance reporting scheme, which is not an accepted method of prevalence calculation, and not all the patients reported to them were included. Their estimated expected concurrence rates were therefore markedly overestimated, and the observed rates underestimated. 

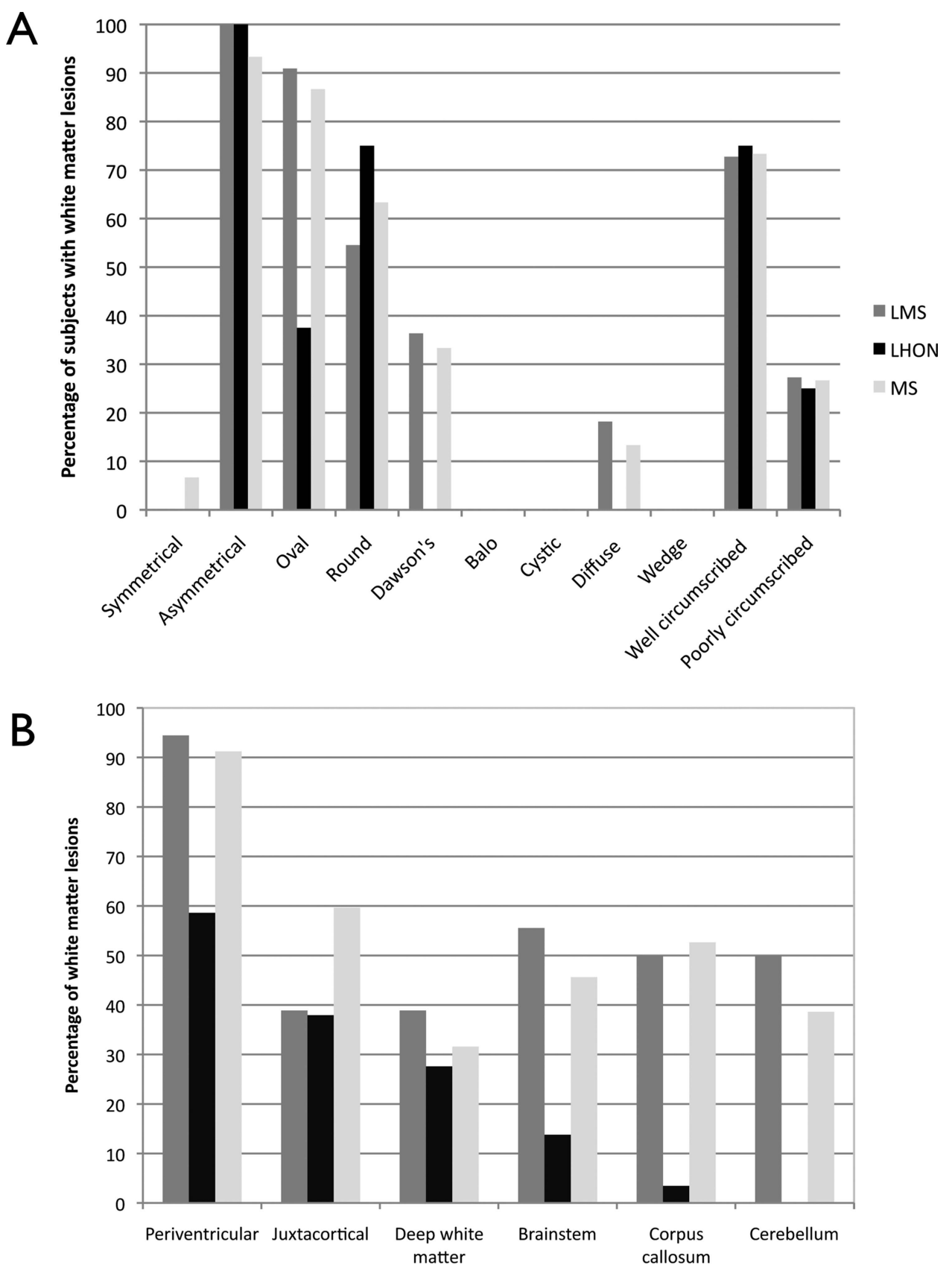

Figure 1 Bar graphs showing (A) morphology of T2 lesions in each participant group (B) lesion location in each participant group. LHON, Leber's hereditary optic neuropathy; LMS, MS-like disease in association with LHON; MS, multiple sclerosis.

The influence of gender is intriguing. The penetrance of LHON in women is much lower than in men. ${ }^{7}{ }^{20}$ However, it appears that once a woman develops LHON clinically, she has a very high risk of developing either radiological or clinical MS. It is not clear whether those with asymptomatic lesions at the time of MRI will develop clinical features in the future, nor what the risk is in asymptomatic female carriers of Leber's mutation.

The limitations of this study include the small numbers of patients with LMS because of its rarity. Also, we did not use quantitative analysis tools to assess MRIs because they were not obtained in a standardised manner and were collected from many different sources. However, the expert reviewers all have extensive experience in MS MRI analysis and there was excellent agreement between their scoring. It was not possible to blind the experts to the presence of optic neuropathy in MRIs; however, this would not have aided them in distinguishing between LHON and LMS. The scan protocols did not include high resolution or fat saturated images of the optic nerves. Owing to the retrospective nature of the data collection, gadolinium-enhanced MRI was also not available for many participants, and therefore not assessed. This would be an interesting area of future study.

We note that more LMS patients were felt to have MRIs typical of MS than fulfilled the revised McDonald criteria for dissemination in space. This is because the experts were asked to judge whether the scan was 'compatible with' rather than diagnostic of MS. This assessment therefore included other features of MS such as highly suggestive lesion morphology including Dawson's fingers or juxtacortical curved $\mathrm{u}$-fibre lesions. 

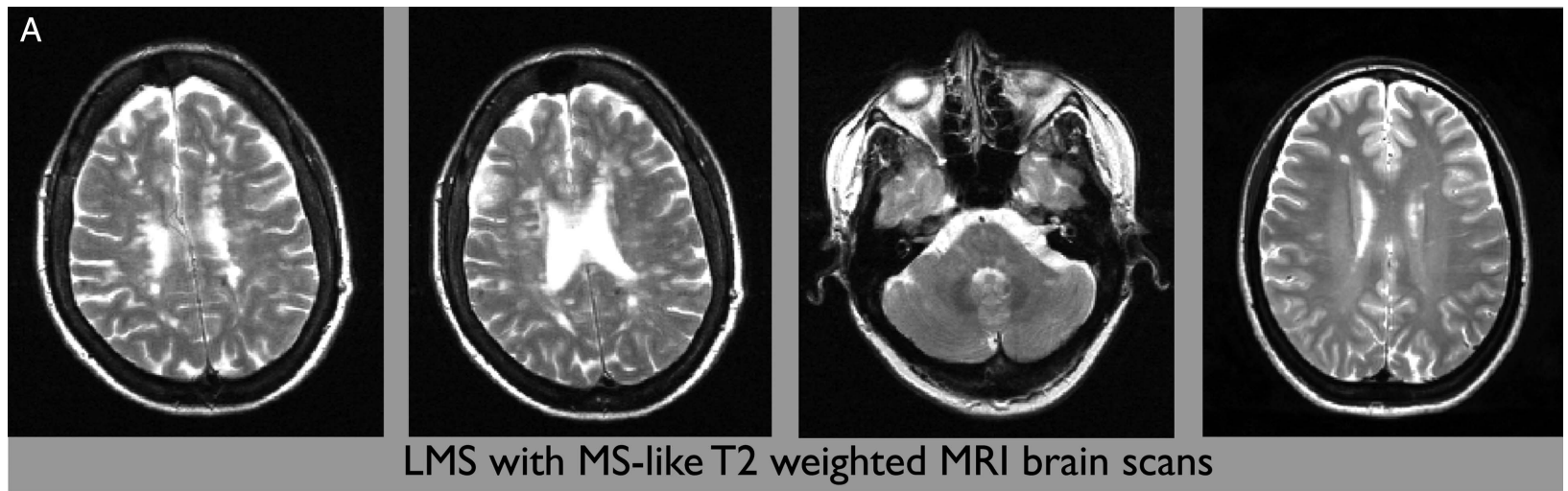

LMS with MS-like T2 weighted MRI brain scans
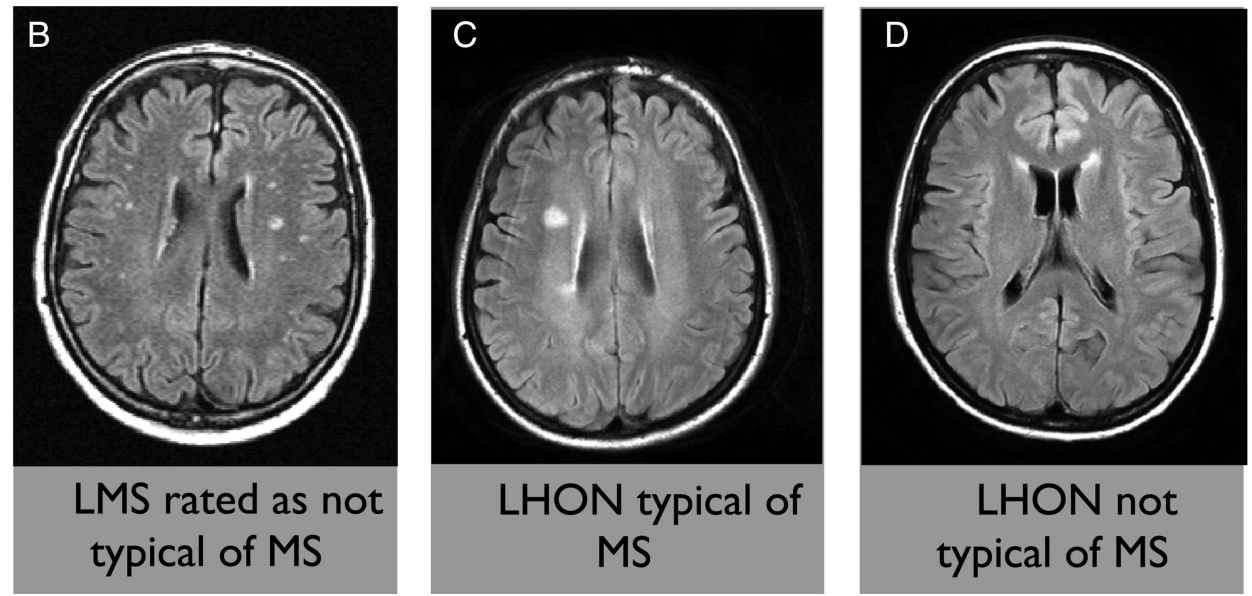

Figure 2 Examples of T2 brain MRI from the study data set. (A) Representative slices of four different patients with LMS (multiple sclerosis (MS)-like disease in association with Leber's hereditary optic neuropathy (LHON)) who were considered to have scans typical of MS. (B) A patient with LMS whose scan was considered by two of three reviewers not to be typical of MS. (C) A patient with LHON and no symptoms of MS who had the brain MRI felt to be typical of MS. (D) A patient with LHON and no symptoms of MS who had white matter lesions not felt to be typical of MS.

This is the first blinded observational study of the MRI features of LHON and LMS. It demonstrates similar conventional brain MRI appearances in MS and LMS. Our study supports the notion that mitochondrial pathways may be important in the development of MS and also demonstrates that being female in association with having LHON confers one of the highest identified risks for MS. It also highlights that MRI should always be used in the context of the clinical picture when making the diagnosis of MS, and that rarer overlap disorders should be considered. Further work on gender and mitochondrial influences is required.

\section{Author affiliations}

${ }^{1}$ Oxford University Hospitals NHS Trust, Oxford, UK

${ }^{2}$ Nuffield Department of Clinical Neurosciences, Oxford University, Oxford, UK

${ }^{3}$ Department of Neurology, Medical University of Graz, Graz, Austria

${ }^{4}$ Department of Radiology, Division of Neuroradiology, Medical University of Graz, Graz, Austria

${ }^{5}$ Department of Radiology, Hospital Vall d'Hebron, Barcelona, Spain

${ }^{6} \mathrm{UCL}$ Institute of Neurology, London, UK

${ }^{7}$ Department of Neurological and Behavioural Sciences, University of Siena, Siena, Italy

${ }^{8}$ Neuroimaging Research Unit and Department of Neurology, San Raffaele Scientific Institute, Vita-Salute San Raffaele University, Milan, Italy

${ }^{9}$ Department of Neurology, Glostrup Hospital, University of Copenhagen, Copenhagen, Denmark

${ }^{10}$ Department of Radiology, St. Josef Hospital Ruhr-University, Bochum, Germany

Acknowledgements The authors would like to thank the members of the MAGNIMS network for their input into this project.

Contributors LM and JP had full access to all of the data in the study and take responsibility for the integrity of the data and the accuracy of the data analysis. JP, WK and LM were involved in the study concept. LM, OC, MTD, MF, JLF, AG, WK,
$C L$, MAR, NDS, AT, TY and JP were involved in the recruitment of patients. CE, FF and $A R$ were involved in the analysis of scans. LM and JP were involved in the analysis of data and manuscript preparation. All authors provided advice for the study design and critically reviewed the paper.

Competing interests LM was funded by the Medical Research Council UK fellowship (G0901996) during the course of this study. CE has received funding for travel and speaker honoraria from Biogen Idec, Bayer Schering Pharma, Merck Serono and Teva Pharmaceutical Industries Ltd/sanofi-aventis; received research support from Merck Serono, Biogen Idec and Teva Pharmaceutical Industries Ltd/sanofi-aventis; and serves on scientific advisory boards for Bayer Schering Pharma, Biogen Idec, Merck Serono, Novartis and Teva Pharmaceutical Industries Ltd/sanofi-aventis. FF serves on scientific advisory boards for Bayer Schering Pharma, Biogen Idec, Merck Serono, Novartis, D-Pharm Ltd and Teva Pharmaceutical Industries Ltd/sanofi-aventis; serves on the editorial boards of Cerebrovascular Diseases, Multiple Sclerosis, the Polish Journal of Neurology and Neurosurgery, Stroke and the Swiss Archives of Neurology and Psychiatry; and has received speaker honoraria from Biogen Idec, Bayer Schering Pharma, Merck Serono and sanofi-aventis. ÀR serves on scientific advisory boards for NeuroTEC Pharma, AdvanceCell Bayer-Schering Pharma, Biogen Idec, Novartis, OLEA Medical and BTG International Ltd, has received speaker honoraria from Bayer Schering Pharma, Sanofi-Aventis, Bracco, Merck-Serono, Teva Pharmaceutical Industries Ltd, Biogen Idec and OLEA Medical; receives research support from Bayer Schering Pharma and has research agreements with Siemens AG. OC serves as a consultant for Biogen, Novartis and General Electric; she is a member of the Editorial Boards of Neurology and Multiple Sclerosis Journal; she receives research support from the UK MS Society, UCL/ UCLH NIHR BRC, CDRC and EPSRC. MF serves on scientific advisory boards for Teva Pharmaceutical Industries and Genmab A/S; has received compensation for consulting services and/or speaking activities from Bayer Schering Pharma, Biogen Idec, Genmab A/S, Merck Serono and Teva Pharmaceutical Industries and receives research support from Bayer Schering Pharma, Biogen Idec, Genmab A/S, Merck Serono, Teva Pharmaceutical Industries, the Italian Ministry of Health, Fondazione Italiana Sclerosi Multipla, Cure PSP and the Jacques and Gloria Gossweiler Foundation (Switzerland). JLF has served on scientific advisory boards and received funding for travel related to these activities as well as honoraria from Biogen Idec, Merck Serono, Sanofi-Aventis, Teva, Novartis and Almirall. She has received speaker honoraria from Biogen Idec, Merck 
Serono and Teva. JLF has also served as an advisor on preclinical development for Takeda. $\mathrm{CL}$ has received consulting fees or speaking fees from Biogen Idec, Bayer Schering Germany, Teva, Genzyme, Sanofi-Aventis and Novartis, as well as grant support from Bayer Schering Germany, Novartis and Merck Serono. MAR has received speaker honoraria from Biogen Idec and Serono Symposia International Foundation and receives research support from the Italian Ministry of Health and Fondazione Italiana Sclerosi Multipla. NDS has served on scientific advisory boards, received speaker honoraria, served as a consultant or received research support from: BioMS Medical, Biogen Idec, Bayer Schering Pharma, Merck Serono, NeuroRx Research, Novartis, Teva Pharmaceutical Industries Ltd and Italian Multiple Sclerosis Society. AT has received honoraria from Sereno Symposia International Foundation and Bayer. TY has received honoraria from Biogen Idec and Novartis. JP has received support for scientific meetings and honoraria for advisory work from Merck Serono, Biogen Idec, Novartis, Teva, Chugai Pharma and Bayer Schering, and unrestricted grants from Merck Serono, Novartis, Biogen Idec and Bayer Schering. Her hospital trust receives funds for her role as clinical lead for the RSS, and she has received grants from the MS society for unrelated research studies.

Ethics approval From local ethics committees to data collection centres.

Provenance and peer review Not commissioned; externally peer reviewed.

Open Access This is an Open Access article distributed in accordance with the Creative Commons Attribution Non Commercial (CC BY-NC 4.0) license, which permits others to distribute, remix, adapt, build upon this work non-commercially, and license their derivative works on different terms, provided the original work is properly cited and the use is non-commercial. See: http://creativecommons.org/ licenses/by-nc/4.0/

\section{REFERENCES}

1 Harding AE, Sweeney MG, Miller DH, et al. Occurrence of a multiple sclerosis-like illness in women who have a Leber's hereditary optic neuropathy mitochondrial DNA mutation. Brain 1992;115(Pt 4):979-89.

2 Palace J. Multiple sclerosis associated with Leber's hereditary optic neuropathy. J Neurol Sci 2009;286:24-7.

3 Vanopdenbosch L, Dubois B, D'Hooghe MB, et al. Mitochondrial mutations of Leber's hereditary optic neuropathy: a risk factor for multiple sclerosis. J Neurol 2000;247:535-43.

4 Riordan-Eva P, Sanders MD, Govan GG, et al. The clinical features of Leber's hereditary optic neuropathy defined by the presence of a pathogenic mitochondrial DNA mutation. Brain 1995;118(Pt 2):319-37.

5 Lees F, Macdonald AM, Turner JW. Leber's disease with symptoms resembling disseminated sclerosis. J Neurol Neurosurg Psychiatry 1964;27:415-21.
6 Kellar-Wood H, Robertson N, Govan GG, et al. Leber's hereditary optic neuropathy mitochondrial DNA mutations in multiple sclerosis. Ann Neurol 1994;36:109-12.

7 Man PY, Griffiths PG, Brown DT, et al. The epidemiology of Leber hereditary optic neuropathy in the North East of England. Am J Hum Genet 2003; 72:333-9.

8 Polman $\mathrm{CH}$, Reingold SC, Banwell B, et al. Diagnostic criteria for multiple sclerosis: 2010 revisions to the McDonald criteria. Ann Neurol 2011;69:292-302.

9 Kuker W, Weir A, Quaghebeur G, et al. White matter changes in Leber's hereditary optic neuropathy: MRI findings. Eur J Neurol 2007;14:591-3.

10 Inglese $M$, Rovaris $M$, Bianchi $S$, et al. Magnetic resonance imaging, magnetisation transfer imaging, and diffusion weighted imaging correlates of optic nerve, brain, and cervical cord damage in Leber's hereditary optic neuropathy. J Neurol Neurosurg Psychiatry 2001;70:444-9.

11 Swanton JK, Fernando K, Dalton CM, et al. Modification of MRI criteria for multiple sclerosis in patients with clinically isolated syndromes. J Neurol Neurosurg Psychiatry 2006;77:830-3.

12 Kovacs GG, Hoftberger R, Majtenyi K, et al. Neuropathology of white matter disease in Leber's hereditary optic neuropathy. Brain 2005;128(Pt 1):35-41.

13 Mahad D, Ziabreva I, Lassmann $\mathrm{H}$, et al. Mitochondrial defects in acute multiple sclerosis lesions. Brain 2008;131(Pt 7):1722-35.

14 Lu F, Selak M, O'Connor J, et al. Oxidative damage to mitochondrial DNA and activity of mitochondrial enzymes in chronic active lesions of multiple sclerosis. J Neurol Sci 2000;177:95-103.

15 Dutta R, McDonough J, Yin X, et al. Mitochondrial dysfunction as a cause of axonal degeneration in multiple sclerosis patients. Ann Neurol 2006;59:478-89.

16 Mahad D, Lassmann H, Turnbull D. Review: mitochondria and disease progression in multiple sclerosis. Neuropathol Appl Neurobiol 2008;34:577-89.

17 Campbell GR, Ziabreva I, Reeve AK, et al. Mitochondrial DNA deletions and neurodegeneration in multiple sclerosis. Ann Neurol 2011;69:481-92.

18 Ban M, Elson J, Walton A, et al. Investigation of the role of mitochondrial DNA in multiple sclerosis susceptibility. PLOS ONE 2008;3:e2891.

19 Chalmers RM, Robertson N, Compston DAS, et al. Sequence of mitochondrial DNA in patients with multiple sclerosis. Ann Neurol 1996;40:239-43.

20 Pfeffer G, Burke A, Yu-Wai-Man P, et al. Clinical features of MS associated with Leber hereditary optic neuropathy mtDNA mutations. Neurology 2013;81:2073-81.

21 Hirst C, Ingram G, Pickersgill T, et al. Increasing prevalence and incidence of multiple sclerosis in South East Wales. I Neurol Neurosurg Psychiatry 2009;80:386-91. 